\title{
Neurological diseases and COVID-19: prospective analyses using the UK Biobank
}

\author{
Nicola Veronese ${ }^{1}$ (D) . Lee Smith $^{2} \cdot$ Mario Barbagallo $^{1} \cdot$ Gianluigi Giannelli $^{3} \cdot$ Maria Gabriella Caruso $^{3}$. \\ Anna Maria Cisternino ${ }^{3} \cdot$ Maria Notarnicola $^{3} \cdot$ Chao Cao $^{4,5} \cdot$ Thomas Waldhoer $^{6} \cdot$ Lin Yang $^{7,8}$
}

Received: 8 March 2021 / Accepted: 23 April 2021 / Published online: 5 May 2021

(c) The Author(s) 2021

\begin{abstract}
COVID-19 (Coronavirus disease-19) may present with neurological signs, but whether people already affected by neurological conditions are at a higher risk of contracting COVID-19 is still not known. We, therefore, aimed to investigate the association of previously diagnosed neurological conditions with COVID-19. 502,536 community-dwelling UK Biobank participants (54.4\% male, mean age 56.6 \pm 10.3 years) were included. Among these, 57,463 participants had a diagnosis of neurological conditions (11.43\%) and a total of 1326 COVID-19-positive cases were identified $(0.26 \%)$. Neurological conditions were identified through medical history and linkage to data on hospital admissions (ICD-10 code G00-G99). COVID-19 presence was diagnosed using the data provided by Public Health England. The association of previous diagnosis of neurological conditions with COVID-19 was evaluated through logistic regressions, adjusted for potential confounders, reported as odds ratios (ORs) with their 95\% confidence intervals (CIs). Nerve, nerve root and plexus disorders (G50-G59) were the most common conditions identified. The presence of COVID-19 was almost doubled in neurological conditions compared to the general population $(0.45$ vs. $0.24 \%, p<0.0001)$. Previously diagnosed neurological conditions were associated with $60 \%$ higher odds of COVID-19 positive in the multivariable-adjusted model ( $\mathrm{OR}=1.6,95 \%$ CI 1.4-1.8). Other degenerative diseases of the nervous system, extrapyramidal and movement disorders, polyneuropathies and other disorders of the peripheral nervous system, cerebral palsy and other paralytic syndromes were significantly associated with a higher odds of COVID-19. The presence of neurological conditions was associated with a significantly higher likelihood of COVID-19 compared to the general population.
\end{abstract}

Keywords COVID-19 · Neurological conditions · UK Biobank

\section{Introduction}

Coronaviruses are common ribonucleic acid viruses. In human beings, these viruses may infect several systems, including respiratory and gastrointestinal systems [1]. Infection by several coronaviruses usually lead to mild,

Nicola Veronese

nicola.veronese@unipa.it

1 Geriatric Unit, Department of Internal Medicine and Geriatrics, University of Palermo, Palermo, Italy

2 The Cambridge Centre for Sport and Exercise Sciences, Anglia Ruskin University, Cambridge, UK

3 National Institute of Gastroenterology "S. de Bellis", Research Hospital, Castellana Grotte, Italy

4 Program in Physical Therapy, Washington University School of Medicine, St. Louis, MO, USA self-limiting upper respiratory tract infections [2]. However, other coronaviruses are associated with severe acute respiratory syndrome (SARS-CoV) and Middle East respiratory syndrome (MERS-CoV).

Neurological manifestations are also being reported, even if they should be considered as atypical presentation

5 Center for Human Nutrition, Washington University School of Medicine, St. Louis, MO, USA

6 Department of Epidemiology, Centre for Public Health, Medical University of Vienna, Vienna, Austria

7 Department of Cancer Epidemiology and Prevention Research, Cancer Control Alberta, Alberta Health Services, Calgary, Canada

8 Departments of Oncology and Community Health Sciences, University of Calgary, Calgary, Canada 
of this infectious disease [3]. The most common neurological manifestations include headache and dizziness, followed by delirium, that often is the first manifestation of COVID19 in older adults [3, 4]. However, similar to other viruses, some literature has reported the presence of cerebrovascular events (such as stroke), Guillain-Barre syndrome, acute transverse myelitis, and acute encephalitis in people affected by COVID-19 [5]. Of importance, it is has been reported that neurological manifestations may precede the typical features of COVID-19 [6]. Finally, the vulnerability of some neurological conditions for severe forms of COVID-19 (and by inference the likelihood of symptomatic COVID19) has already been demonstrated in various studies $[3,7$, 8]. Therefore, all these studies suggest that the association between COVID-19 and neurological conditions could be significant.

At the same time, very little is known regarding the risk of COVID-19 in people already affected by neurological conditions. People having these medical conditions can be at a higher risk of COVID-19 since they are more frail [9] and they usually have more severe impairment in their immune system [10] than in the general population. Understanding whether people with neurological conditions are at higher risk of COVID-19 could be of importance to better recognise the presence of this novel infectious disease in neurological settings, and finally, protect these people from its possible sequelae.

Given this background, the present study aimed to investigate the risk of COVID-19 in people with previous diagnosis of neurological conditions compared to the general population in the UK Biobank, a large study involving more than 500,000 participants.

\section{Materials and methods}

\section{Study population}

This research is covered by generic ethical approval from the NHS Research Ethics Committee (Ref. 11/NW/0382) for UK Biobank research. The analyses presented here were approved within project 41,245 by the UK Biobank research committee on 03 May 2019. The data used for the aims of this paper were originally collected from 2007 [11]. Briefly, the UK Biobank is a large health-focused resource, which specifically aims to understand the importance of environmental factors, genetics and lifestyle impact upon a broad array of health outcomes. The recruitment phase was conducted through a formal mail invitation, sent to 9.2 million households. Of them, more than 500,000 individuals attended UK Biobank assessment centres, to provide informed consent and complete baseline assessments. This process included touchscreen questionnaires, in-person interviews, and physical health examinations (full details for the UK Biobank's assessment processes are available elsewhere) [11].

\section{Exposure: neurological conditions}

Participants in the UK Biobank living with neurological conditions were identified based on medical history and linkage to data on hospital admissions. Since June 2013, data on UK Biobank participants' primary/main diagnoses were extracted from all their hospital inpatient records and coded according to the International Classification of Disease version-10 (ICD-10). In January 2019, data on participants' secondary diagnoses were also extracted from their hospital inpatients records and coded according to the ICD10. Both data fields were used to identify overall and specific neurological conditions as follows: G00-G09: inflammatory disease of the central nervous system; G10-G14: systemic atrophies primarily affecting the central nervous system; G20-G26: extrapyramidal and movement disorders; G30-G32: other degenerative diseases of the nervous system; G35-G37: demyelinating diseases of the central nervous system; G40-G47: episodic and paroxysmal disorders; G50-G59: nerve, nerve root and plexus disorders; G60-G65: polyneuropathies and other disorders of the peripheral nervous system; G70-G73: diseases of myoneural junction and muscle; G80-G83: cerebral palsy and other paralytic syndromes; G89-G99: other disorders of the nervous system. Details of the algorithms used to combine the data from different sources to identify primary or secondary diagnosis have been described previously and are freely available on the UK Biobank website (www.ukbiobank.ac.uk).

\section{COVID-19 diagnosis}

Baseline data from UK Biobank were linked to COVID19 test results provided by Public Health England [12, 13], including the specimen date, origin (whether the person was an inpatient or not) and result (positive or negative). Confirmed COVID-19 was defined as at least one positive test result. Data were available for the period 16th March 2020 to 25th May 2020. The specimen were collected from an acute (emergency) care provider, an A\&E department, an inpatient location, or resulted from health care associated infection, therefore, including symptomatic patients requiring hospital admission, or general inpatient screening, which includes asymptomatic patients.

\section{Covariates}

A range of sociodemographic, lifestyle behaviour factors and medical conditions were included as covariates. Data were extracted on age (continuous), sex (male, female), 
BMI (normal, overweight, obese, missing), household income (less than $£ 18,000, £ 18,000$ to $£ 30,999, £ 31,000$ to $£ 51,999, £ 52,000$ to $£ 100,000$, greater than $£ 100,000$, missing), country of birth (UK, oversea, missing), and ethnicity (White, mixed, Asian or Asian British, Black or Black British, Chinese, others, missing). Information on age, sex, income, and country of birth were collected using a computerised questionnaire at UK Biobank assessment centres. BMI was calculated by body weight $(\mathrm{kg}) /$ height (metre) $)^{2}$, both of which were measured by a research assistant during physical health assessments.

In agreement with the American Heart Association (AHA) guidelines [14], the present study calculated a dietary score to assess healthy dietary behaviour. Briefly, for constructing this score, the UK Biobank participants completed a questionnaire on their habitual dietary intake. To determine a categorical healthy diet variable for the purposes of our analysis, the UK Biobank's dietary intake data on the consumption of fruit, vegetables, fish and processed and red meats were extracted. A healthy diet was defined as adherence to at least two of the healthy food items that include total fruit and vegetable intake, total fish intake, and a low intake of processed and red meat [14]. Physical activity was assessed with the International Physical Activity Questionnaires (IPAQ) short form [15]. Based on the IPAQ scoring system, physical activity levels were defined as low, moderate, and high in the UK Biobank. Smoking status was defined in the UK Biobank as non-, past, and current smokers; likewise, alcohol intake was defined as non-, past, and current drinkers. In addition, previous diagnosis of medical conditions were considered as presence of diabetes, cancer, fractures, or other (other than diabetes, cancer, fracture) serious medical condition/disability.

\section{Statistical analysis}

Participant characteristics including neurological condition were summarised using means and standard deviation for age, and frequencies and percentages for other variables. The distributions of participant characteristics were compared by neurological condition status using linear regression for continuous variables and Chi square tests for categorical variables. Sample size and prevalence of neurological conditions in the UK Biobank were presented for all and each specific neurological condition. In addition, proportions of specific neurological conditions among overall neurological conditions were presented.

Univariate, age-adjusted and multivariable-adjusted logistic regressions were carried out to estimate associations between existing neurological conditions and positive COVID-19 test results. Multivariable models adjusted for age, sex, BMI, household income, country of birth, dietary score, physical activity, smoking and alcohol drinking behaviour, and other medical conditions. Covariates with missing data were included in the model as missing categories. Furthermore, specific associations of eleven neurological conditions with positive COVID-19 test results were estimated using univariate, age-adjusted and multivariableadjusted logistic regression models. In each neurological condition specific model, participants with the other ten neurological conditions were removed from the analysed sample to avoid biased estimations. All statistical analyses were conducted in Stata 14.0 (StataCorp, Texas, USA).

\section{Results}

In total, 502,536 UK Biobank participants (54.4\% male, mean age $56.6 \pm 10.3$ years) were included in this analysis. Among these, 57,463 participants had a diagnosis of neurological conditions (11.43\%), and a total of 1326 COVID19 -positive cases were identified $(0.26 \%)$.

As shown in Table 1, participants with neurological conditions, compared with those without prior neurological conditions, were more likely to be obese ( $34.9 \%$ vs. $23.0 \%)$, had household income less than $£ 18,000$ (27.3\% vs. $18.3 \%)$, had low level of physical activity ( $18.4 \%$ vs. $14.7 \%)$, currently smoke (13.1\% vs. $10.2 \%)$ and had other medical conditions (53.8\% vs. $32.4 \%)$. The adherence to a healthy dietary score was significantly higher in people without neurological conditions. The proportion of participants who tested positive for COVID-19 were $0.24 \%(n=1066)$ and $0.45 \%(n=260)$ in those without and with neurological conditions, respectively ( $p<0.0001$ for all these comparisons).

Table 2 shows the sample sizes for the overall and each specific neurological condition as primary or secondary conditions in the UK Biobank study population prior to the COVID pandemic. The most common neurological condition was nerve, never root and plexus disorders $(n=24,984$, $43.5 \%$ of all neurological conditions, and $4.97 \%$ of the UK Biobank study population), followed by episodic and paroxysmal disorder $(n=20,915,36.4 \%$ of all neurological conditions, and $4.16 \%$ of the UK Biobank study population), which comprised over $70 \%$ of all neurological conditions. Other specific neurological conditions were generally around or below $5 \%$ of all diagnoses.

Table 3 presents the associations of neurological condition diagnoses with COVID-19-positive results in the UK Biobank. Living with neurological conditions was associated with nearly a twofold higher odds of COVID-19 positivity in the univariate $(\mathrm{OR}=1.9,95 \% \mathrm{CI} 1.7-2.2)$ and age-adjusted $(\mathrm{OR}=1.9,95 \%$ CI 1.7-2.2) models, which remained significant in the multivariable-adjusted model $(\mathrm{OR}=1.6,95 \%$ CI 1.4-1.8) after adjusting for age, sex, BMI, household income, country of birth, dietary score, physical activity smoking and alcohol drinking behaviour and other medical 
Table 1 Sample characteristics of participants diagnosed with neurological conditions in the UK Biobank study (data reported in percentages, unless otherwise noted)

\begin{tabular}{|c|c|c|c|c|}
\hline & \multirow[t]{2}{*}{ Total $(n=502,536)$} & \multicolumn{2}{|c|}{ Neurological condition diagnoses } & \multirow[t]{2}{*}{$P$ values } \\
\hline & & No $(n=445,073)$ & Yes $(n=57,463)$ & \\
\hline Age, years ${ }^{\mathrm{a}}$ & $56.6(10.3)$ & $56.4(10.3)$ & $58.1(10.3)$ & $<0.001$ \\
\hline Sex & & & & 0.888 \\
\hline Male & 54.4 & 54.4 & 54.3 & \\
\hline Female & 45.6 & 45.5 & 45.7 & \\
\hline Body mass index & & & & $<0.001$ \\
\hline Normal & 32.8 & 33.9 & 24.4 & \\
\hline Overweight & 42.2 & 42.6 & 39.1 & \\
\hline Obese & 24.3 & 23.0 & 34.9 & \\
\hline Missing & 0.6 & 0.5 & 1.6 & \\
\hline Household income & & & & $<0.001$ \\
\hline Less than $£ 18,000$ & 19.3 & 18.3 & 27.3 & \\
\hline$£ 18,000-£ 30,999$ & 21.5 & 21.4 & 22.2 & \\
\hline$£ 31,000-£ 51,999$ & 22.0 & 22.5 & 18.5 & \\
\hline$£ 52,000-£ 100,000$ & 17.2 & 17.9 & 11.2 & \\
\hline Greater than $£ 100,000$ & 4.6 & 4.9 & 2.3 & \\
\hline Missing & 15.4 & 15.0 & 18.5 & \\
\hline Country of birth & & & & $<0.001$ \\
\hline UK & 91.7 & 91.6 & 92.5 & \\
\hline Oversea & 7.9 & 8.1 & 7.1 & \\
\hline Missing & 0.4 & 0.3 & 0.4 & \\
\hline Ethnicity & & & & $<0.001$ \\
\hline White & 94.1 & 94.0 & 94.2 & \\
\hline Mixed & 0.6 & 0.6 & 0.6 & \\
\hline Asian or Asian British & 4.6 & 1.6 & 1.5 & \\
\hline Black or Black British & 2.0 & 2.0 & 1.9 & \\
\hline Chinese & 0.3 & 0.3 & 0.2 & \\
\hline Others & 0.9 & 0.9 & 0.9 & \\
\hline Missing & 0.5 & 0.6 & 0.7 & \\
\hline Dietary score ${ }^{\mathrm{b}}$ & & & & $<0.001$ \\
\hline 0 & 7.5 & 7.4 & 8.2 & \\
\hline 1 & 31.7 & 31.7 & 31.9 & \\
\hline 2 & 42.4 & 42.6 & 41.2 & \\
\hline 3 & 18.2 & 18.2 & 18.5 & \\
\hline Missing & 0.2 & 0.2 & 0.2 & \\
\hline Physical activity (IPAQ) & & & & $<0.001$ \\
\hline Low & 15.2 & 14.7 & 18.5 & \\
\hline Moderate & 32.6 & 33.1 & 29.0 & \\
\hline High & 32.3 & 32.7 & 28.9 & \\
\hline Missing & 19.9 & 19.5 & 23.6 & \\
\hline Smoking behaviour & & & & $<0.001$ \\
\hline Non-smoker & 54.4 & 55.2 & 48.2 & \\
\hline Past smoker & 34.4 & 34.0 & 37.8 & \\
\hline Current smoker & 10.5 & 10.2 & 13.1 & \\
\hline Missing & 0.6 & 0.6 & 0.9 & \\
\hline Alcohol drinking behaviour & & & & $<0.001$ \\
\hline Non-drinker & 4.5 & 4.3 & 6.0 & \\
\hline Past drinker & 3.6 & 3.2 & 6.5 & \\
\hline Current drinker & 91.6 & 92.2 & 87.0 & \\
\hline Missing & 0.3 & 0.3 & 0.5 & \\
\hline
\end{tabular}


Table 1 (continued)

\begin{tabular}{lllll}
\hline & Total $(n=502,536)$ & \multicolumn{2}{l}{ Neurological condition diagnoses } & $P$ values \\
& & No $(n=445,073)$ & Yes $(n=57,463)$ & \\
\hline Medical condition $^{c}$ & 65.2 & & & $<0.001$ \\
No & 34.8 & 67.6 & 46.2 & \\
Yes & & 32.4 & 53.8 & $<0.001$ \\
COVID positive & 0.26 & 0.24 & 0.45 & \\
Yes & & & \\
\hline
\end{tabular}

${ }^{a}$ Reported data: mean (standard deviation)

${ }^{b}$ Dietary score was derived based on the American Heart Association guideline, range 0-4, with higher score indicates healthier diet

${ }^{\mathrm{c}}$ Including diabetes, cancer, fracture, and any other serious medical conditions/disability

Table 2 Sample size and prevalence of specific neurological conditions in the UK biobank (total $n=502,536$ )

\begin{tabular}{|c|c|c|c|}
\hline & $n^{\mathrm{a}}$ & $\begin{array}{l}\% \text { In neurological } \\
\text { conditions }^{\mathrm{a}}\end{array}$ & $\begin{array}{l}\% \text { In overall } \\
\text { population }^{\mathrm{a}}\end{array}$ \\
\hline Overall & 57,463 & 100 & 11.43 \\
\hline \multicolumn{4}{|l|}{ Specific neurological condition } \\
\hline Inflammatory diseases of the central nervous system (G00-G09) & 918 & 1.6 & 0.18 \\
\hline Systemic atrophies primarily affecting the central nervous system (G10-G14) & 643 & 1.1 & 0.13 \\
\hline Extrapyramidal and movement disorders (G20-G26) & 3340 & 5.81 & 0.66 \\
\hline Other degenerative diseases of the nervous system (G30-G32) & 1969 & 3.43 & 0.39 \\
\hline Demyelinating diseases of the central nervous system (G35-G37) & 2032 & 3.54 & 0.40 \\
\hline Episodic and paroxysmal disorders (G40-G47) & 20,915 & 36.4 & 4.16 \\
\hline Nerve, nerve root and plexus disorders (G50-G59) & 24,984 & 43.5 & 4.97 \\
\hline Polyneuropathies and other disorders of the peripheral nervous system (G60-G65) & 3027 & 5.27 & 0.60 \\
\hline Diseases of myoneural junction and muscle (G70-G73) & 766 & 1.33 & 0.15 \\
\hline Cerebral palsy and other paralytic syndromes (G80-G83) & 3491 & 6.08 & 0.69 \\
\hline Other disorders of the nervous system (G89-G99) & 6317 & 10.99 & 1.26 \\
\hline
\end{tabular}

${ }^{\text {a }}$ Proportions of specific neurological conditions add up higher than the overall due to neurological conditions

conditions (for full results of logistic regression, see Supplementary Table 1).

To evaluate whether this association may vary between different types of neurological condition diagnoses, logistic regression models were carried out for each specific neurological condition (for specific OR and 95\% CI, see Table 3 and Fig. 1). In the multivariable-adjusted models, other degenerative diseases of the nervous system (G30-G32) (multivariable-adjusted $\mathrm{OR}=6.3,95 \% \mathrm{CI} 4.4$ to 9.0 ) were associated with sixfold higher odds of positive COVID19 test results. In addition, extrapyramidal and movement disorders (G20-G26) (multivariable-adjusted $\mathrm{OR}=3.2$, 95\% CI 2.2-4.7), polyneuropathies and other disorders of the peripheral nervous system (G60-G65) (multivariableadjusted $\mathrm{OR}=2.9,95 \%$ CI 2.0-4.3), and cerebral palsy and other paralytic syndromes (G80-G83) (multivariableadjusted $\mathrm{OR}=2.9,95 \%$ CI 2.2-4.2) were associated with approximately threefold higher odds of positive COVID-19 test results. In multivariable-adjusted logistic regression models of aforementioned neurological conditions, $p$ values remained significant after Bonferroni correction for multiple testing (data not shown). These associations were either non-significant or with ORs smaller than 2 for other specific neurological conditions.

\section{Discussion}

In this large prospective study carried out using UK Biobank participants, living with previously diagnosed neurological conditions was associated with $60 \%$ higher odds of COVID19-positive testing. The higher odds of COVID-19 were particularly significant in some neurological conditions, including extrapyramidal and movement disorders, polyneuropathies, and paralysis/cerebral palsy. 
Table 3 Univariate, age-adjusted and multivariable-adjusted associations of neurological conditions with COVID-19-positive diagnosis in the UK Biobank (total $n=502,536$ )

\begin{tabular}{|c|c|c|c|c|c|c|c|c|c|}
\hline \multirow[b]{2}{*}{ Overall } & \multicolumn{3}{|c|}{ Univariate } & \multicolumn{3}{|c|}{ Age-adjusted $^{\mathrm{a}}$} & \multicolumn{3}{|c|}{$\begin{array}{l}\text { Multivariable- } \\
\text { adjusted }^{\mathrm{b}}\end{array}$} \\
\hline & 1.9 & $(1.7-2.2)$ & $<0.001$ & 1.9 & $(1.7-2.2)$ & $<0.001$ & 1.6 & $(1.4-1.8)$ & $<0.001$ \\
\hline \multicolumn{10}{|l|}{ Specific neurological condition $^{c}$} \\
\hline $\begin{array}{l}\text { Inflammatory diseases of the central nervous system (G00- } \\
\text { G09) }\end{array}$ & 2.3 & $(0.9-5.5)$ & 0.067 & 2.3 & $(1.0-5.6)$ & 0.064 & 2.0 & $(0.8-4.7)$ & 0.136 \\
\hline $\begin{array}{l}\text { Systemic atrophies primarily affecting the central nervous } \\
\text { system (G10-G14) }\end{array}$ & 2.0 & $(0.6-6.1)$ & 0.248 & 2.0 & $(0.6-6.3)$ & 0.228 & 1.8 & $(0.6-5.6)$ & 0.315 \\
\hline Extrapyramidal and movement disorders (G20-G26) & 3.5 & $(2.4-5.1)$ & $<0.001$ & 3.7 & $(2.5-5.4)$ & $<0.001$ & 3.2 & $(2.2-4.7)$ & $<0.001$ \\
\hline Other degenerative diseases of the nervous system (G30-G32) & 7.1 & $(5.0-10.1)$ & 0.000 & 7.5 & $(5.3-10.7)$ & $<0.001$ & 6.3 & $(4.4-9.0)$ & $<0.001$ \\
\hline $\begin{array}{l}\text { Demyelinating diseases of the central nervous system (G35- } \\
\text { G37) }\end{array}$ & 1.6 & $(0.8-3.3)$ & 0.161 & 1.6 & $(0.8-3.3)$ & 0.171 & 1.6 & $(0.8-3.2)$ & 0.215 \\
\hline Episodic and paroxysmal disorders (G40-G47) & 1.9 & $(1.5-2.3)$ & $<0.001$ & 1.9 & $(1.5-2.3)$ & $<0.001$ & 1.5 & $(1.2-1.9)$ & $<0.001$ \\
\hline Nerve, nerve root and plexus disorders (G50-G59) & 1.5 & $(1.2-1.9)$ & $<0.001$ & 1.5 & $(1.2-1.9)$ & $<0.001$ & 1.3 & $(1.1-1.6)$ & 0.013 \\
\hline $\begin{array}{l}\text { Polyneuropathies and other disorders of the peripheral nervous } \\
\text { system (G60-G65) }\end{array}$ & 3.7 & $(2.6-5.5)$ & $<0.001$ & 3.9 & $(2.6-5.7)$ & $<0.001$ & 2.9 & $(2.0-4.3)$ & $<0.001$ \\
\hline Diseases of myoneural junction and muscle (G70-G73) & 1.6 & $(0.5-5.1)$ & 0.394 & 1.7 & $(0.5-5.2)$ & 0.377 & 1.3 & $(0.4-4.2)$ & 0.613 \\
\hline Cerebral palsy and other paralytic syndromes (G80-G83) & 3.7 & $(2.6-5.3)$ & $<0.001$ & 3.8 & $(2.7-5.5)$ & $<.001$ & 2.9 & $(2.0-4.2)$ & $<0.001$ \\
\hline Other disorders of the nervous system (G89-G99) & 1.5 & $(1.0-2.2)$ & 0.082 & 1.5 & $(1.0-2.3)$ & 0.071 & 1.2 & $(0.8-1.9)$ & 0.323 \\
\hline
\end{tabular}

${ }^{a}$ Models adjusted for age only

${ }^{b}$ Models adjusted for age, sex, BMI, household income, country of birth, ethnicity, dietary score (based on the American Heart Association recommendation), physical activity, smoking behaviour, alcohol drinking behaviour, and medical conditions including diabetes, cancer, fracture, and any other serious medical conditions/disability

${ }^{\mathrm{c}}$ Models for each specific neurological condition excluded other neurological conditions

People with neurological diseases had a higher proportion of obesity, were poorer, but had higher proportion of people being physically active and a better attitude in healthier lifestyle, even if they were more frequently smokers than their counterparts, making them at higher risk of COVID-19 infection. To the best of our knowledge, this is the first study to report a prospective association of neurological conditions with COVID-19. The association between COVID-19 and neurological disorders appears strong, as suggested by the recent literature. For example, data from 214 patients with COVID-19 reported neurological symptoms in at least one third of the patients [6]. The data reported so far suggests that COVID-19 infected patients with more severe systemic presentations were more likely to have neurologic symptoms, compared with those with milder forms of the infection [6]. Neurological conditions, by themselves, have a great epidemiological impact [16] and they are associated with a high risk of disability [17] and poor quality of life [18]. At the same time, the infection from COVID-19 may further worsen the clinical course of these conditions and possibly lead to higher mortality risk.

In the present study, data suggests that some specific neurological conditions are more likely to be affected by COVID-19. In particular, compared to people free of neurological conditions, the odds of SARS-CoV-2 infection were three times higher, after considering potential confounders, in people affected by extrapyramidal and movement disorders, a category that included Parkinson's disease. Some studies, contrary to our findings, reported that there is no evidence, or lack of evidence, suggesting that patients with Parkinson's disease may have a similar risk of contracting COVID-19 to general population [19, 20]. However, the small sample sizes and the retrospective design of these studies limit these findings.

In addition, the present study found that COVID-19 was more prevalent in people affected by polyneuropathies and other disorders of the peripheral nervous system than in the general population. Again, the association between COVID19 and these specific neurological conditions is intriguing. In an observational study of 214 patients with confirmed diagnoses of COVID-19, approximately $10 \%$ presented with peripheral nervous system symptoms [21]. These symptoms may include hypogeusia, hyposmia, hypoplasia and neuralgia that now are increasingly recognised as highly specific for COVID-19 [22]. Moreover, the category of polyneuropathies and other disorders of the peripheral nervous system included Guillain-Barré Syndrome which may be a consequence not only of COVID-19, but also of other viral infections [23]. Finally, the present study reported a significant higher odds of COVID-19 in participants affected 


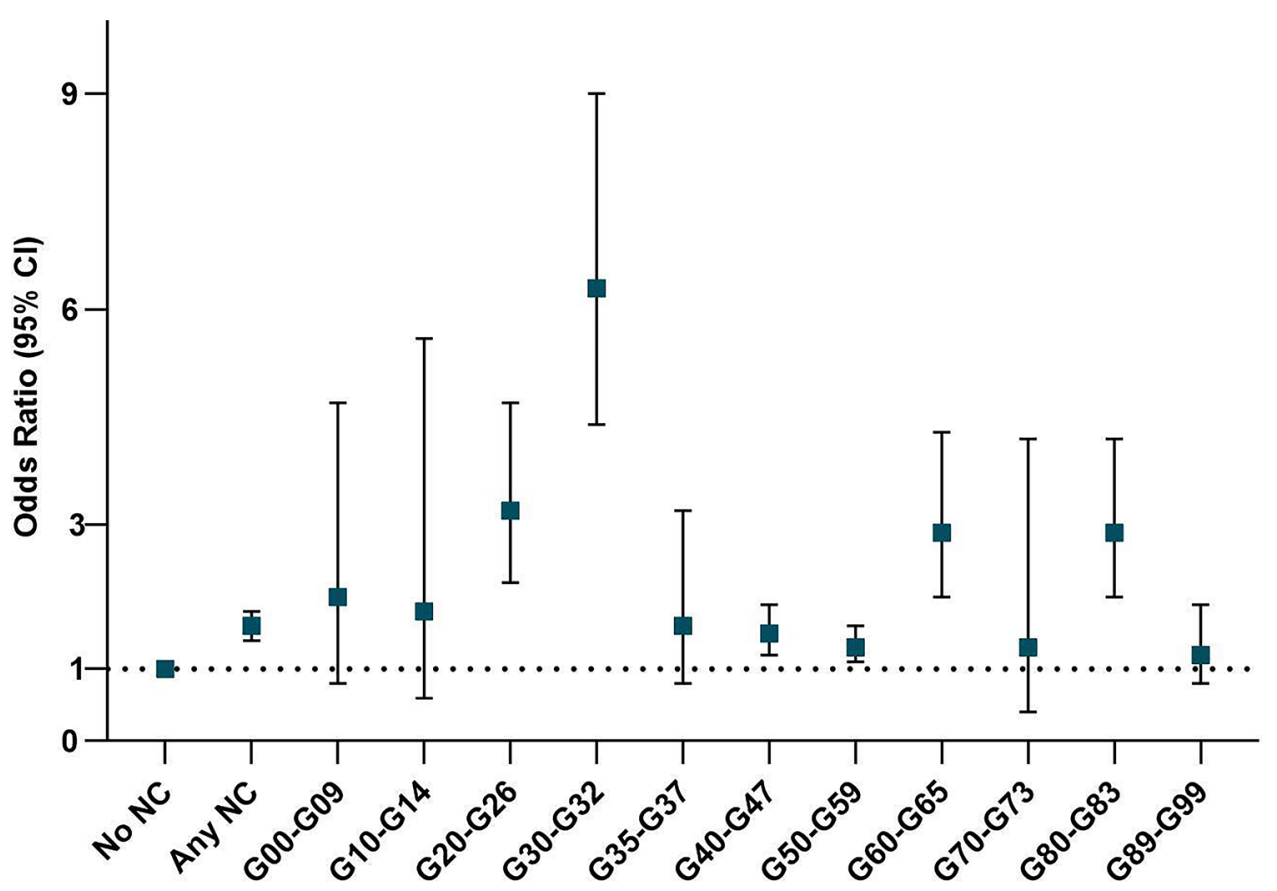

Fig. 1 Multivariable-adjusted odds ratios of neurological conditions with COVID-19-positive diagnosis in the UK Biobank (total $n=502,536)^{\mathrm{a}, \mathrm{b}}$. NC Neurological condition, G00-G09 Inflammatory diseases of the central nervous system, G10-G14 Systemic atrophies primarily affecting the central nervous system, G20-G26 Extrapyramidal and movement disorders, G30-G32 Other degenerative diseases of the nervous system, G35-G37 Demyelinating diseases of the central nervous system, $G 40-G 47$ Episodic and paroxysmal disorders, G50-G59 Nerve, nerve root and plexus disorders, G60-G65 Polyneuropathies and other disorders of the peripheral nervous sys-

by cerebral palsy and other paralytic syndromes comparing to those free of neurological conditions. To the best of our knowledge, this is the first report to highlight this association. In this already "at risk" population, COVID-19 may have significant detrimental consequences in comparison to the general population. Further research to confirm or refute the present findings and to further explain potential underlying mechanisms is warranted.

The present study has several strengths and limitations. The strengths of the UK Biobank include its extensive phenotyping which enables the adjustment for demographic and lifestyle risk factors, disease and ill health, and its large sample size [13]. However, it is not representative of the general population living in the UK. For example, it has been estimated that the overall prevalence of COVID-19 in the United Kingdom is $0.55 \%$ [24], whilst we found an overall presence of $0.26 \%$. Nevertheless, the prospective nature of the UK Biobank study was able to document neurological condition diagnoses prior to the COVID-19 pandemic, facilitating certain levels of temporal association. Moreover, we did not include information regarding medications. For example, many neurological diseases require immunosuppressive tem, G70-G73 Diseases of myoneural junction and muscle, G80-G83 Cerebral palsy and other paralytic syndromes, G89-G99 Other disor-

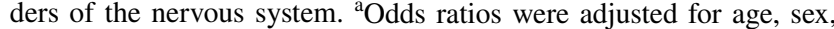
BMI, household income, country of birth, dietary score (based on the American Heart Association recommendation), physical activity, smoking behaviour, alcohol drinking behaviour, and medical conditions including diabetes, cancer, fracture, and any other serious medical conditions/disability. ${ }^{b}$ Odds ratios for each specific neurological condition excluded other neurological conditions

medications that are obviously associated with more severe forms of COVID-19. Furthermore, the COVID-19 testing was not implemented in the overall population. However, the criteria of COVID-19 testing in the UK are primarily based on symptoms. Given this, we are unable to distinguish whether higher likelihood of SARS-CoV-2 infection being symptomatic versus asymptomatic in certain neurological conditions or a higher susceptibility to acquiring the infection from people affected by neurological diseases. Finally, the observed higher COVID-19 positivity in neurological diseases could be due to higher likelihood of SARS-CoV-2 infection being symptomatic versus asymptomatic in certain neurological conditions, such as extrapyramidal and movement disorders, polyneuropathies and paralysis/cerebral palsy. Alternatively, our observed association might have been due to a greater susceptibility of COVID-19 among people with neurological conditions because they may experience limited self-cleanliness and other aspects of grooming behaviour, poor adherence to social distancing or shielding and other aspects of social behaviour associated with nursing home residence, carer visits, and reliance on public transport. 
In conclusion, this study shows that the people affected by neurological conditions have a significantly higher likelihood of COVID-19 in comparison to the general population. In particular, this investigation highlights that people with some common specific neurological conditions (such as neurological paralyses, polyneuropathies, and extrapyramidal disorders) may be at a greater risk of COVID-19. These findings need to be confirmed in future studies. Given the added neurological manifestations associated with COVID-19, the prognosis of neurological patients with COVID-19 warrants further investigation.

Supplementary Information The online version contains supplementary material available at https://doi.org/10.1007/s13760-021-01693-3.

Funding Open access funding provided by Università degli Studi di Palermo within the CRUI-CARE Agreement.

\section{Declarations}

Conflict of interest The authors declare that they have no conflicts of interest.

Research involving human participants and/or animals Approved by NHS Research Ethics Committee (Ref. 11/NW/0382).

Informed consent Requested to all participants.

Open Access This article is licensed under a Creative Commons Attribution 4.0 International License, which permits use, sharing, adaptation, distribution and reproduction in any medium or format, as long as you give appropriate credit to the original author(s) and the source, provide a link to the Creative Commons licence, and indicate if changes were made. The images or other third party material in this article are included in the article's Creative Commons licence, unless indicated otherwise in a credit line to the material. If material is not included in the article's Creative Commons licence and your intended use is not permitted by statutory regulation or exceeds the permitted use, you will need to obtain permission directly from the copyright holder. To view a copy of this licence, visit http://creativecommons.org/licenses/by/4.0/.

\section{References}

1. Chen Y, Liu Q, Guo D (2020) Emerging coronaviruses: genome structure, replication, and pathogenesis. J Med Virol 92(4):418-423

2. Zumla A, Chan JF, Azhar EI, Hui DS, Yuen K-Y (2016) Coronaviruses - drug discovery and therapeutic options. Nat Rev Drug Discov 15:327

3. Ahmad I, Rathore FA (2020) Neurological manifestations and complications of COVID-19: a literature review. J Clin Neurosci. https://doi.org/10.1016/j.jocn.2020.05.017

4. Soysal P, Kara O (2020) Delirium as the first clinical presentation of the coronavirus disease 2019 in an older adult. Psychogeriatrics. https://doi.org/10.1111/psyg.12587

5. Zhou Y, Li W, Wang D, Mao L, Jin H, Li Y, Hong C, Chen S, Chang J, He Q (2020) Clinical time course of COVID-19, its neurological manifestation and some thoughts on its management. Stroke Vasc Neurol 5(2):1
6. Mao L, Jin H, Wang M, Hu Y, Chen S, He Q, Chang J, Hong C, Zhou Y, Wang D (2020) Neurologic manifestations of hospitalized patients with coronavirus disease 2019 in Wuhan, China. JAMA Neurol 77(6):683-690

7. Ahmed MU, Hanif M, Ali MJ, Haider MA, Kherani D, Memon GM, Karim AH, Sattar A (2020) Neurological manifestations of COVID-19 (SARS-CoV-2): a review. Front Neurol 11:518

8. Correia AO, Feitosa PWG, de Sousa Moreira JL, Nogueira SÁR, Fonseca RB, Nobre MEP (2020) Neurological manifestations of COVID-19 and other coronaviruses: a systematic review. Neurol Psychiatry Brain Res. https://doi.org/10.1016/j.npbr.2020.05.008

9. Cigolle CT, Ofstedal MB, Tian Z, Blaum CS (2009) Comparing models of frailty: the health and retirement study. J Am Geriatr Soc 57:830-839

10. Ransohoff RM, Schafer D, Vincent A, Blachère NE, Bar-Or A (2015) Neuroinflammation: ways in which the immune system affects the brain. Neurotherapeutics 12:896-909

11. Biobank UK (2007) The UK Biobank: rationale, design and development of a large-scale prospective resource. UK Biobank Coordinating Centre, Stockport

12. Armstrong J, Rudkin J, Allen N, Crook D, Wilson D, Wyllie D (2020) Dynamic linkage of COVID-19 test results between public health England's second generation surveillance system and UK Biobank. Microb Genomics 6(7):1

13. Hastie CE, Mackay DF, Ho F, Celis-Morales CA, Katikireddi SV, Niedzwiedz CL, Jani BD, Welsh P, Mair FS, Gray SR (2020) Vitamin D concentrations and COVID-19 infection in UK Biobank. Diabetes Metab Syndr Clin Res Rev 14(4):1

14. Rutten-Jacobs LC, Larsson SC, Malik R, Rannikmäe K, Sudlow CL, Dichgans M, Markus HS, Traylor M, Consortium ISG (2018) Genetic risk, incident stroke, and the benefits of adhering to a healthy lifestyle: cohort study of 306473 UK Biobank participants. BMJ 363:k4168

15. Hagströmer M, Oja P, Sjöström M (2006) The international physical activity questionnaire (IPAQ): a study of concurrent and construct validity. Public Health Nutr 9:755-762

16. Pringsheim T, Fiest K, Jette N (2014) The international incidence and prevalence of neurologic conditions: how common are they? Neurology 83:1661-1664

17. Kayes NM, Schluter PJ, McPherson KM, Taylor D, Kolt GS (2009) The physical activity and disability survey-revised (PADS-R): an evaluation of a measure of physical activity in people with chronic neurological conditions. Clin Rehabil 23:534-543

18. Calvert M, Pall H, Hoppitt T, Eaton B, Savill E, Sackley C (2013) Health-related quality of life and supportive care in patients with rare long-term neurological conditions. Qual Life Res 22:1231-1238

19. Prasad S, Holla VV, Neeraja K, Surisetti BK, Kamble N, Yadav R, Pal PK (2020) Parkinson's disease and COVID-19: perceptions and implications in patients and caregivers. Mov Disord 35(6):912-914

20. Papa SM, Brundin P, Fung VS, Kang UJ, Burn DJ, Colosimo C, Chiang HL, Alcalay RN, Trenkwalder C, Committee M-SI (2020) Impact of the COVID-19 pandemic on Parkinson's disease and movement disorders. Mov Disord 6(10): 1002

21. Xu X-W, Wu X-X, Jiang X-G, Xu K-J, Ying L-J, Ma C-L, Li S-B, Wang H-Y, Zhang S, Gao H-N (2020) Clinical findings in a group of patients infected with the 2019 novel coronavirus (SARS-Cov-2) outside of Wuhan, China: retrospective case series. BMJ 368:1

22. Vaira LA, Salzano G, Deiana G, De Riu G (2020) Anosmia and ageusia: common findings in COVID-19 patients. Laryngoscope. https://doi.org/10.1002/lary.28692

23. Alberti P, Beretta S, Piatti M, Karantzoulis A, Piatti ML, Santoro P, Viganò M, Giovannelli G, Pirro F, Montisano DA (2020) 
Guillain-Barré syndrome related to COVID-19 infection. Neurol Neuroimmunol Neuroinflammation 7:1

24. Iacobucci G (2020) Covid-19: prevalence has quadrupled in England since start of September, study shows. Br Med J Publ Group. https://doi.org/10.1136/bmj.m3850
Publisher's Note Springer Nature remains neutral with regard to jurisdictional claims in published maps and institutional affiliations. 